\title{
Surgical Inking and Double Embedding Technique for Routine Histopathology to Save Time and Effort
}

\author{
Savitri M Nerune*, Namrata B Mestri, Anil Reddy Konduru, Neha Kathpal \\ Balasaheb R Yelikar and Ankur Kumar
}

Department of Pathology, Shri B. M. Patil Medical College Hospital and Research Centre, BLDE University, Vijayapur, Karnataka, India

\begin{abstract}
Background: Routine histopathological specimens like appendix and fallopian tube require individual processing, block making and slide preparation. This process is not only time consuming and requires more effort but also takes up more space during archiving. The objective of the present study was to use commercially available different acrylic colors to ink specimen of appendix and fallopian tube of different patients, process them in a single cassette and preparation of single paraffin block and slide.
\end{abstract}

Methods: 100 cases of appendix and fallopian tubes were taken to evaluate ink mapping technique using Fevicrylß Acrylic colours. Blocks and slides were prepared, labelling on the slides was done using the chromacodes method for identification of the specimen. These slides were then reported by the pathologist.

Result: There was a $21 \%$ reduction in the amount of paraffin required for preparation of a double embedded block in comparison to two paraffin blocks by conventional method. It was also noted that there was $18 \%$ reduction in volume as compared to two conventional paraffin blocks.

Conclusion: This method of using surgical inking technique not only brings down the effort and time required, but also storage space and the costs involved along with faster identification and reporting compared to the conventional method.

\section{Keywords: Surgical inking, Fevicryl@ Acrylic Colours, Double-Embedding, Chromacode}

\section{Introduction}

Inking of borders of radical specimens to know involvement by tumour tissue is the most widely used technique in cases of malignancy. ${ }^{[1-3]}$ Inking also helps in identifying a specimen, its orientation and this process can be colour coded for uniformity. There are many traditionally used marking inks available in the market like India ink, alcian blue, plain gelatine, gelatine with added colours, tissue marking dye and acrylic colours. Acrylic colors have many advantages as compared to others in terms of cost effectiveness, availability, reproducibility of results and ease of application. ${ }^{[4-6]}$ India ink imparts a single colour whereas acrylic colors are available in different shades of colors where it can be easily applied for identifying various surgical margins of radical specimen in cases of malignancy. ${ }^{[2]}$

For routine histopathology specimens, for example in case of appendix and fallopian tube, multiple sections are taken and embedded in paraffin blocks then slides are prepared for each. However, after the reporting of these cases the blocks and slides are to be archived and preserved for a period of 10 years and maintaining this requires large amount of storage space and resources. Few authors have used the technique of inking and double embedding of specimens in a single paraffin block. ${ }^{[7,8]}$

The objective of the present study was to analyze commercially available different acrylic colors to ink specimens of appendix and fallopian tube of different patients and process them in a single cassette and further preparation of single paraffin block and slide to reduce the efforts, time for sectioning and to save the storage space.

\section{Materials and Methods}

We chose 100 cases of appendix and fallopian tubes to evaluate ink mapping technique used by Kini $\mathrm{U}$ et al. ${ }^{[7]}$ In this, they used ink mapping technique for orientation of mucosal biopsies in frozen section to routine histopathology processing technique. To avoid any unforeseen consequences, already reported specimens of appendix and fallopian tubes were chosen for the study. We used the Fevicryl ${ }^{\circledR}$ Acrylic colours, which were found to be best possible alternatives for the costly surgical inks.

After identifying the specimen and noting down the patient details, two colours, Pearl Black (306) and Pearl Green (304) were chosen. Once the colour was assigned for a patient, it was documented using the chromacodes. 
[9] Black colour was assigned Chromacode as [------], and green colour was assigned as $[+++++]$ (Fig 1). The specimens having an even histopathology ID serial numbers were given green colour and odd ones were given black colour. Inking of resected specimens of appendix and fallopian tube were done with the help of paint brushes and were allowed to dry. The brushes were washed thoroughly after each application. After drying of the ink, two tissue sections of appendix from different patients inked with different colours were incorporated into one cassette. Same was followed for the fallopian tube sections.

Blocks and slides were prepared, labelling on the slides was done using the same chromacodes method for identification of the specimen. The specimen identification was not a problem, appendix sections while embedding in the paraffin block were easily identified, as the colours were clearly visible and were not washed out after the tissue processing (Fig. 2). Reporting of these slides was done by a pathologist who was blinded to the earlier diagnosis and later, the results were compared to the earlier diagnosis using the conventional histopathology processing technique.

Inked sections were scored based on visibility on microscopy between 1-4, with score 1 allotted for poor visibility, 2 for good, 3 for very good and 4 for excellent visibility.

\section{Results}

A total of 100 cases were included in the study. The microscopic diagnoses of the cases $(n=100)$ were compared with the diagnoses given previously. The diagnosis was matching in all of the cases and there were no problems due to missing colour on the sections. Black and green colors were clearly visible on microscopy in all sections of appendix and fallopian tube (Fig.3).

The mean size of an individual block in case of routine histopathology processing method was $2.19 \mathrm{~cm} \times 1.82 \mathrm{~cm}$ $\mathrm{x} 1.65 \mathrm{~cm}$ having a volume of $6.6 \mathrm{~cm}^{3}$, while the blocks prepared with the double embedding technique measured $2.94 \mathrm{~cm} \times 2.15 \mathrm{~cm} \times 1.71 \mathrm{~cm}$ with a volume of $10.8 \mathrm{~cm}^{3}$. Although there appears to be a marginal increase in dimensions in the double embedded blocks, when they were compared to two blocks prepared by conventional processing, the reduction in volume was noted to be approximately $18 \%$. The mean weight of the two blocks in case of conventional processing was 10.2 gm whereas a single double embedded block weighed $8.1 \mathrm{gm}$, amounting to an approximately $21 \%$ reduction in the paraffin used.

Table 1: Score for Pearl Black and Pearl Green (based on visibility on microscopy).

\begin{tabular}{|c|c|c|c|}
\hline SCORE & PEARL BLACK (50 CASES) & PEARL GREEN (50 CASES) & TOTAL \\
\hline 1 Poor & - & - & 05 \\
\hline 2 Good & - & 05 & 10 \\
\hline 3 Very Good & 05 & 35 & 80 \\
\hline 4 Excellent & 45 & 50 & 100 \\
\hline TOTAL & $\mathbf{5 0}$ & 50 \\
\hline
\end{tabular}

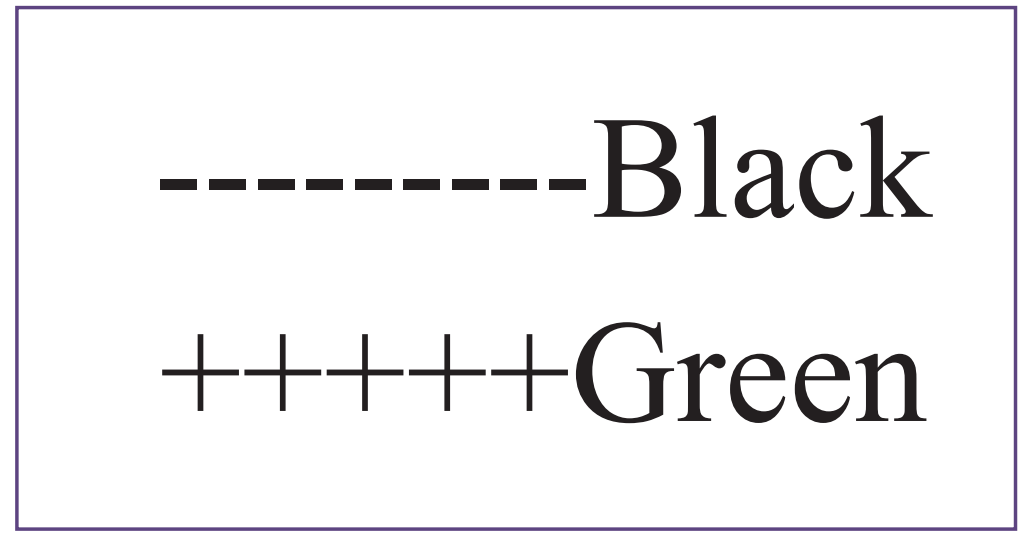

Fig. 1: Inking Chromacodes. 


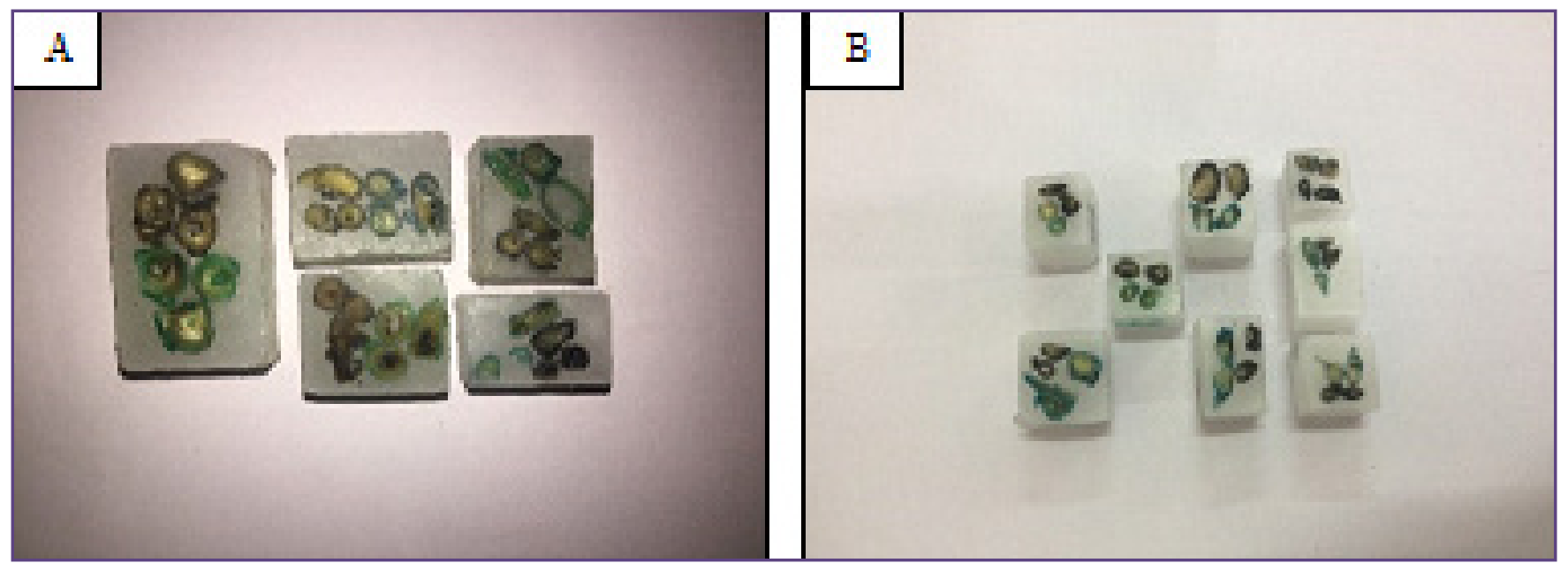

Fig. 2: Shows inked tissues of appendix and fallopian tube from different patients in a single paraffin block. (A and B).

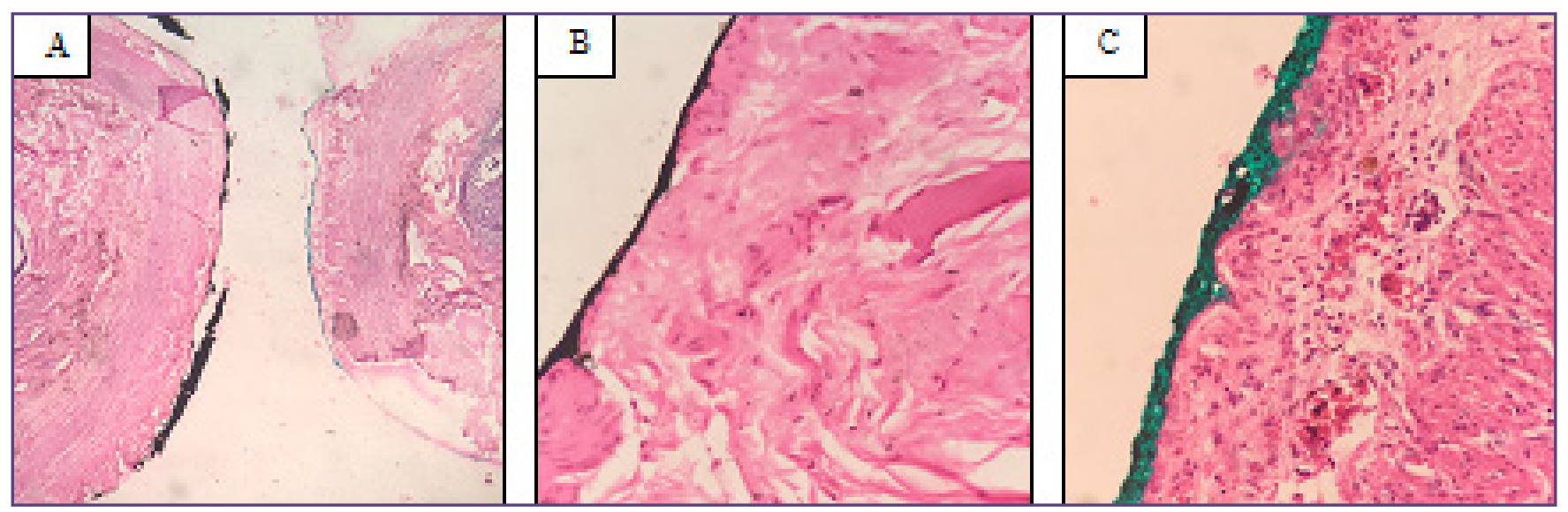

Fig. 3: Microphotographs show sections of appendix and fallopian tube with black and green colors (H\& E 100X A, B, C).

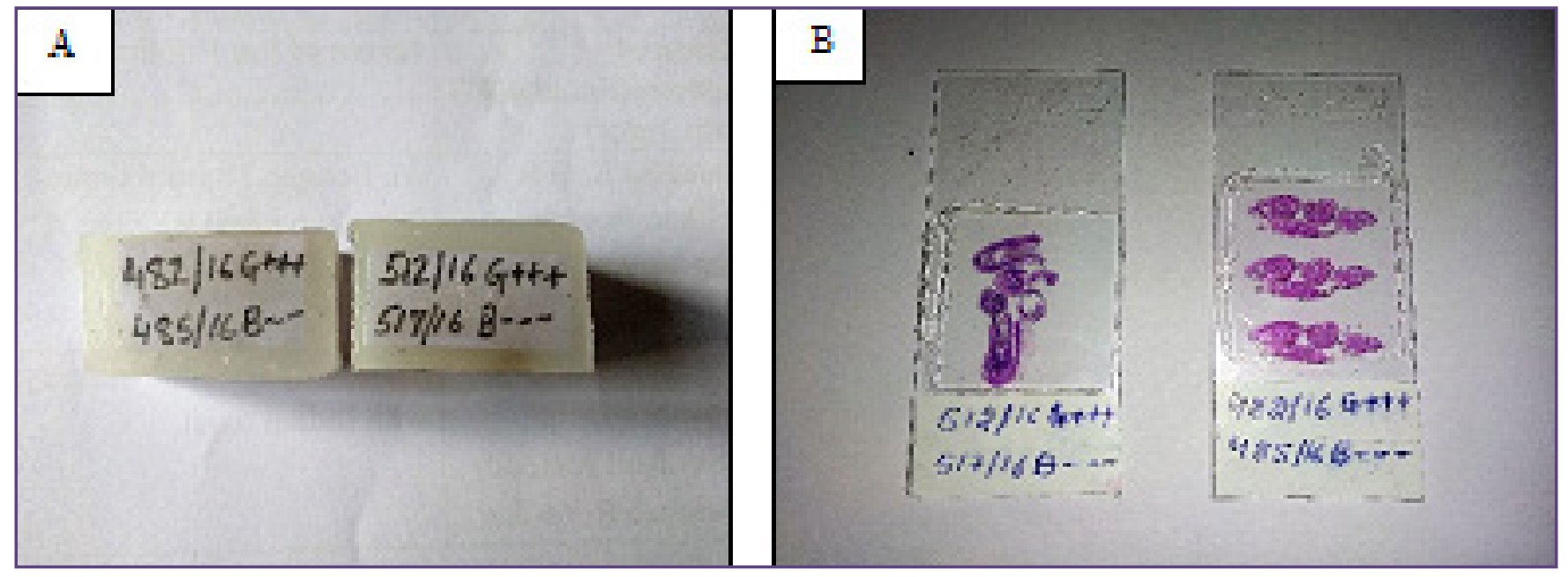

Fig. 4: Shows chromacodes labelling on the paraffin blocks (A) and slides (B). 


\section{Discussion}

Inking of the specimens have been documented to be started by the Egyptians. The application of inking in the form of India ink, surgical inks and recent application of economical $\&$ easily available hobby colours like Fevicryl $®$ are in use now. ${ }^{[1,2]}$ Inking of the specimens in the form of India ink has been used widely since fourth century B.C. ${ }^{\left[{ }^{6]}\right.}$ This ink is made of carbon black molecules in colloidal suspension which after drying forms a waterproof layer. Acrylic colours, pigment suspended in acrylic polymer emulsion used since 20th century have been used increasingly now because of its multiple benefits. They are water resistant, dry faster, easy to apply, cost effective, long lasting and available in multiple colours. In view of these benefits of acrylic colours, we have chosen them over others for this study. ${ }^{[1-6]}$

Inking of the specimens is being widely practiced in routine processing of the tumours for margin clearance in oncology service laboratories. Various authors have used it to improve the diagnostic yield and identification of margin involvement. Tampi $\mathrm{C}$ et al. have demonstrated the utility of the Fevicryl ${ }^{\circledR}$ colours for this purpose replacing the costly surgical inks. With ever increasing workloads and to improve the utilisation and achieving near $100 \%$ efficiency of human resources, the application of this technique has a potential that needs to be studied.

As the procedure of inking for radical specimens is already being followed in the department, there was not much of a difference in applying the colours except for the documentation of the Chromacodes. The time taken for inking each specimen was not more than a couple of minutes. The total standing time after inking, before the specimen was submitted for fixation was on an average 15-20 minutes. Pearl Black and Pearl Green were chosen as these colours offered good visibility and contrast on paraffin blocks and microscopy respectively.

Scoring was done based on the visibility of coloured inks on microscopy ranging from excellent to poor. Out of total 100 cases, score 4 was given for 80 cases, score 3 in 15 cases and score 2 in 5 cases. It was noted that Pearl Black ink offered much better visibility on microscopy than the Pearl Green ink (Table 1).

With the standardization of the technique using the said colours and chromacodes the slide labelling and block labelling were done (Fig. 4). Charts showing the same were made available in the gross room, embedding room and also in the reporting room.

The storage aspect of the slides and blocks for archival purpose were so rewarding and encouraging when the extra time and procedure for the coding is considered. Coming to the economics of the slides, paraffin, effective utilisation of the cassettes for processing specimens using the same resources is also a very good incentive to make this as a routine practice.

\section{Conclusion}

This method can be established as a standard protocol for routine histopathology specimens as it helps in faster identification and quick reporting. It also brings down the cost and time required for making multiple blocks and slides and in turn reduces the space required for archiving them.

\section{Reference}

1. Pursnani D, Arora S, Katyayani P, Ambica C, Yelikar B. Inking in surgical pathology: does the method matter? a procedural analysis of a spectrum of colours. Turk J Pathol. 2016.

2. Sarode SC, Sarode GS, Patil S, Mahajan P, Anand R, Patil A. Comparative Study of Acrylic Color and India Ink for their use as a surgical margin inks in oral squamous cell carcinoma. World J Dent. 2015; 6:26-30.

3. Chiam HW, Maslen PG, Hoffman GJ. Marking the surgical margins of specimens: Commercial acrylic pigments are reliable, rapid and safe. Pathology. 2003; 35:204-6.

4. Shinde V, Phelan C, Gater W, Thomas J. Inking a specimen without the mess. J Clin Pathol. 2008; 61:783.

5. Williams AS, Dakin Haché K. Variable fidelity of tissuemarking dyes in surgical pathology. Histopathology. 2014;64:896-900.

6. Tampi C. In search of the rainbow: Colored inks in surgical pathology. Indian J Pathol Microbiol. 2012; 55:154-7.

7. Kini U, Yadav L, Thomas S. Improvised double-embedding technique of minute biopsies: A mega boon to histopathology laboratory. Indian J Pathol Microbiol. 2015; 58(1):12.

8. Blewitt ES, Pogmore T, Talbot IC. Double embedding in agar/paraffin wax as an aid to orientation of mucosal biopsies. J Clin Pathol. 1982;35:365.

9. Vidimos A, Poblete-Lopez C, Gasbarre C. Mohs surgery. Philadelphia, Pa.: Saunders; 2011.

*Corresponding author:

Dr Savitri M Nerune, Department of pathology, Shri B. M. Patil Medical College, BLDE University Solapur road, Vijayapur-586103, Karnataka, India. Phone: +91 9008179806

Email: nervunesavitri.mallikarjun@bldeuniversity.ac.in

Financial or other Competing Interests: None. 